\title{
In vivo safety evaluations of electrospun nanofibers for biomedical applications
}

\author{
B. Balusamy, A. Senthamizhan, T. Uyar \\ Bilkent University, Ankara, Turkey
}

\subsection{Introduction}

Electrospinning has emerged as one of the most attractive processing techniques to produce nanoscale fibers that can closely mimic the extracellular matrix (ECM) components and to stimulate the natural functionalization of the cells. Electrospun nanofibers of various natural and synthetic polymers were extensively investigated for their potential in biomedical and pharmaceutical applications especially for skin tissue engineering, wound healing, and controlled drug delivery. The nanofibers have unique properties including high surface area and enhanced cellular interactions and protein absorption for facilitating binding sites of cell receptors. They have been highlighted as promising materials for cartilage, bone, vascular, bladder, and tissue engineering applications [1]. To date, numerous clinical applications of electrospun nanofibers are being considered, and thereby, concerns on the safety of these nanofibrous membranes are significantly increased. The nanotoxicology research is mainly foreseeable to address the requirements of regulatory agencies including US Food and Drug Administration (US FDA) and also biosafety needs. Cipitria et al. [2] reviewed and presented the biocompatibility characteristics of poly( $\varepsilon$-caprolactone) (PCL) electrospun nanofibers.

Biocompatibility is generally defined as the compatible nature of any foreign agents with living system or tissue not causing any toxic effects and immunologic rejection and physiologically reactive. The biocompatibility evaluation of a material is generally critical due to their variations in intended use, nature of body, time, and duration. The biocompatible material should not cause any adverse effects including local or systemic, genotoxicity, and developmental toxicity either directly or due to their leachable chemicals. Therefore, the safety of a material that is intended for human use should be systematically investigated in accordance to well-established standard testing guidelines to fulfill the requirements regulatory agencies.

The biological evaluation of a material that is intended for human use shall take part in structured biological evaluation program within a risk management process according to the International Organization for Standardization (ISO) ISO 14971:2007 [3], which particularly addresses the risks associated with medical devices. The ISO (10993-1:2010) [4] standard addresses the biological evaluation 
of medical devices with a series of testing guidelines to deal with a range of possible short-term effects such as acute toxicity; irritation in the skin, eye, and mucous membranes; thrombogenicity; and hemolysis. And also, the standard addresses long-term/specific toxic effects such as subchronic and chronic effects, sensitization, genotoxicity, tumorigenicity, and teratogenicity using various preclinical studies. Following the successful completion of preclinical evaluations, clinical studies in two main types (i) clinical trials (interventional studies) and (ii) observational are conducted in human volunteers to add medical knowledge, which should be in compliance with regulatory agency.

Although numerous in vitro studies have been devoted to investigate the safety of nanofibrous membranes, the rationale of investing in vivo safety studies is comparatively lesser, which obviously slows down further the progress of bedside research $[5,6]$. However, the true effects of a testing material cannot be proved only through animal models, and clinical studies will play vital role in conforming the findings in humans. This chapter highlighted about the in vivo studies conducted on electrospun nanofibers that are used for various applications and their toxicity outcomes. And also, the chapter discusses about the clinical trials conducted using electrospun nanofibers. We made an effort to provide overview on both cases rather than presenting an in-depth literature survey.

\subsection{Safety evaluations of nanofibers}

\subsubsection{In vivo studies in animal models}

The exceptional characteristics of electrospun nanofibrous membrane show an excellent in vitro performance in terms of cell attachment, spreading, and differentiation. The reason for the tremendous growth of electrospun scaffolds in biomedical applications might be due to three main variables: (1) fiber diameter, (2) chemistry, and (3) fiber orientation. However, although electrospun materials show excellent cell and tissue response, a major drawback is their poor mechanical properties, thought to be critical to the success of the surgical repair of tendons. Hakimi et al. [7] developed a bonding technique that enables the processing of electrospun sheets into multilayered, robust, implantable fabrics. The tissue reaction to the patch after implantation in a rat's shoulder is assessed. This animal study was performed to demonstrate safety rather than efficacy of the layered design. Preliminary in vivo evaluation of the assembled patch was carried out for periods of $1,2,4,6$, and 12 weeks.

Fig. 6.1 shows the proposed possible mechanism by which the scaffold may be integrated in vivo, based on gross and histological observation. During the first 4 weeks, it is proposed that cells surrounding the scaffold respond to the morphology of the electrospun layer to align along the nanofibers. At the next phase, around 4-6 weeks, the woven component is increasingly integrated by the tissue. Foreign-body giant cell numbers peak at week 4 , as the electrospun component degrades, and are gradually replaced by more organized cell arrays. At the last time point, at week 12, the remaining scaffold is tightly embedded in dense tissue, inflammation is resolved, and the surrounding tissue begins to remodel. Importantly, it 


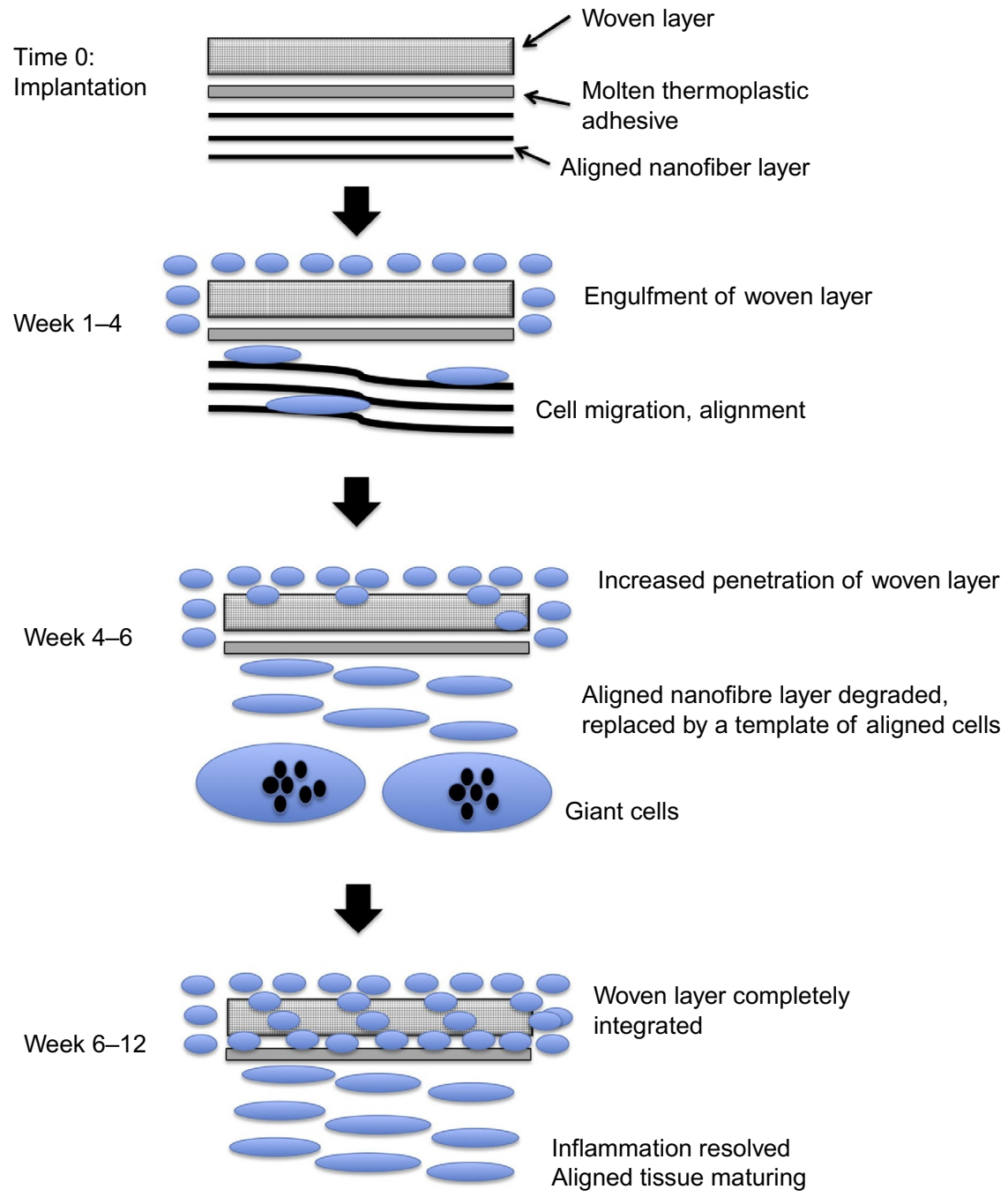

Fig. 6.1 A schematic representation of tissue reaction to the scaffold based on in vivo observations.

Reproduced with permission from Hakimi O, Mouthuy PA, Zargar N, Lostis E, Morrey M, Carr A. A layered electrospun and woven surgical scaffold to enhance endogenous tendon repair. Acta Biomater 2015;26:124-35, with permission from Elsevier.

should be acknowledged that this proposed mechanism is an interpretation of biocompatibility data collected in a rat model. The efficacy reported in the study is based on the animal model, and it is certain that rate and true efficacy cannot be proved in an animal model, and a clinical trial will be vital to confirm our findings in humans. These compelling results warrant further research and development of the scaffold for human application. 
The bare electrospun fibrous membrane, for example, bacterial cellulose (BC), has received considerable interest as wound-dressing material. The insufficient antimicrobial activity is considered to be a critical skin-barrier function in wound healing. The additional active agents have been introduced to solve this problem so far. The strong antimicrobial activities of silver nanoparticles (AgNPs) against many different bacteria, fungi, and viruses have been known for long time. The work has been initiated to incorporate the AgNPs into the bare electrospun nanofibrous membrane. However, the adhesion of anchored nanoparticles is usually very weak. In such a case, the release of nanoparticles is quite normal, and it could cause the cellular toxicity. It has been already well reported that silver is a recognized cause of argyrosis and argyria, and high-level exposure of silver nanoparticles is toxic to mammalian cells [8].

Tseng et al. [9] developed biodegradable vancomycin-loaded poly[lactic-co-glycol acid] (PLGA) nanofibrous membranes for vancomycin release in the cerebral tissue of rats. Following the results from in vitro drug release study, the biodegradable nanofibrous membranes embedded with vancomycin were then placed into the brain surface of rats after operative craniectomy was performed, and the characteristics of vancomycin release from the membranes in vivo were investigated. Fig. 6.2 indicated the gross wound

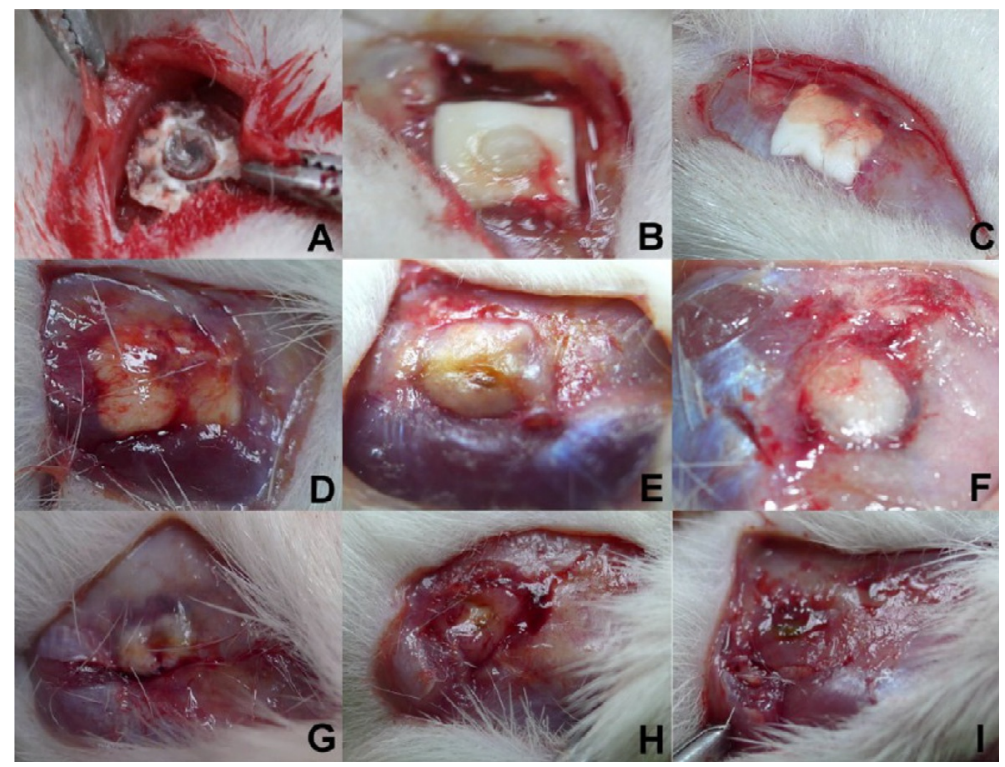

Fig. 6.2 Gross wound conditions: (A) 3 days, (B) 1 week, (C) 2 weeks, (D) 3 weeks, (E) 4 weeks, (F) 5 weeks, (G) 6 weeks, (H) 7 weeks, and (I) 8 weeks. The scalp wound and brain tissue were quite clear, and no infection (exudate, pus, or granulation formation) was noticed during the whole experimental period. By week 8, the PLGA/vancomycin nanomembranes were nearly completely dissolved.

Reproduced with permission from Tseng YY, Kao YC, Liao JY, Chen WA, Liu SJ.

Biodegradable drug-eluting poly[lactic-co-glycol acid] nanofibers for the sustainable delivery of vancomycin to brain tissue: in vitro and in vivo studies. ACS Chem Neurosci 2013;4:

1314-21, with permission from American Chemical Society. 
condition and gradual degradation of the vancomycin-loaded biodegradable PLGA nanofibrous membrane. The scalp wounds and brain tissues were clear, and no infection (exudate, pus, or granulation formation) was noticed grossly. The biodegradable PLGA/ vancomycin nanofibrous membranes degraded gradually after operative implantation and were almost completely diminished by 8 weeks. The histological examination also showed no obvious inflammation reaction of the brain tissues.

Lowe et al. [10] prepared a novel acrylonitrile (AN)-based terpolymers and adopted electrospinning method to prepare nonwoven sheets of bandage/wound-dressing-type materials for storage and delivery applications of nitric oxide (NO) for wound healing, which release NO upon contact to a humidified or physiological environment that binds and releases NO. Fig. 6.3 shows the digital images of NO-treated and control-treated wounds from days 0,7 , and 14 . No significant adverse effects on surrounding skin or wound margins were noted following the treatment with NO or control dressings. Further, the histopathology examinations indicated that the average capillary density was significantly higher at $\sim 60 \%$ for the NO-treated wounds as compared with control-treated wounds. They further suggested that daily bandage changes will significantly enhance healing progression as soon as 4 days. In this novel, NO dressings are easy to apply and change, thus making multiple applications each week possible.

Day 0
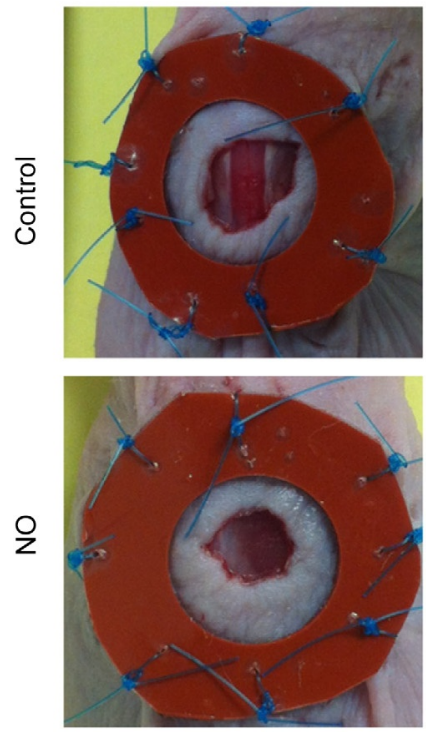

Day $7 \%$ healing
Day 7
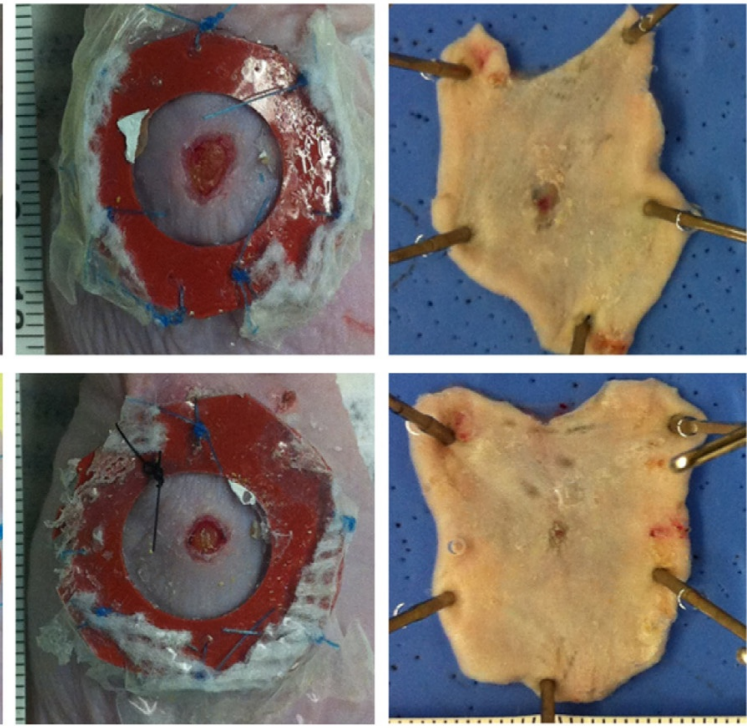

Day $14 \%$ healing

Day 14

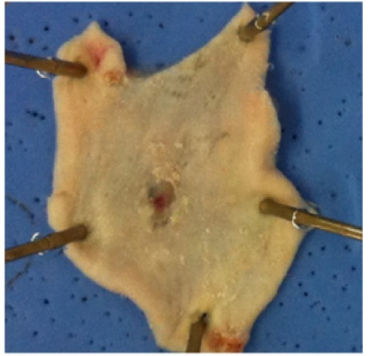

Fig. 6.3 Weekly administration of NO-releasing dressings improves wound closure.

Representative digital images of NO and control wounds at days 0, 7, and 14 in the first study. Images are presented at equivalent scale.

Reproduced with permission from Lowe A, Bills J, Verma R, Lavery L, Davis K, Balkus KJ. Electrospun nitric oxide releasing bandage with enhanced wound healing. Acta Biomater 2015;13: 121-30, with permission from Elsevier. 
The study demonstrated the compatibility of NO of incorporating the electrospun nanofibers in breached skin of the mouse.

The insufficient concentration and nonspecific distribution of the antitumor drugs are the major limitations of the conventional tumor chemotherapy. In the recent past, research has been shown that biodegradable electrospun nanofibers are considered as very promising drug carriers for localized and controlled delivery of antitumor drugs. This might be due the following reasons: biodegradable carriers capable of undergoing gradual decomposition into carbon dioxide, water, and finally disappearing in vivo; maximization of local drug concentration in the immediate tumor environment and minimization of nontarget organ toxicity; delayed release of the drugs at cytotoxic concentrations that are sustained for longer periods at much lower total doses than can be achieved with typical systemic chemotherapeutic regimes; increase in the duration of tumor exposure to the drug; reduction of the frequency of drug administration; and convenience of being cut to almost any size and fabricated into other shapes using different target geometries for clinical applications [11,12]. Liu et al. [11] demonstrated the possibility and feasibility of nanofiber-based local drug delivery system and low-cost therapeutic option supplementary to currently limited clinical protocols for patients with hepatocellular carcinoma (HCC) or secondary hepatic carcinoma (SHCC). The therapeutic process of nanofibers in the treatment of mice is shown in Fig. 6.4.

The doxorubicin hydrochloride (Dox) was chosen as model of antitumor drug due to their broad spectrum antitumor activity and fluorescent property suitable for in vivo tracing. The in vivo antitumor efficacy of Dox fibers was studied in Balb/c mice
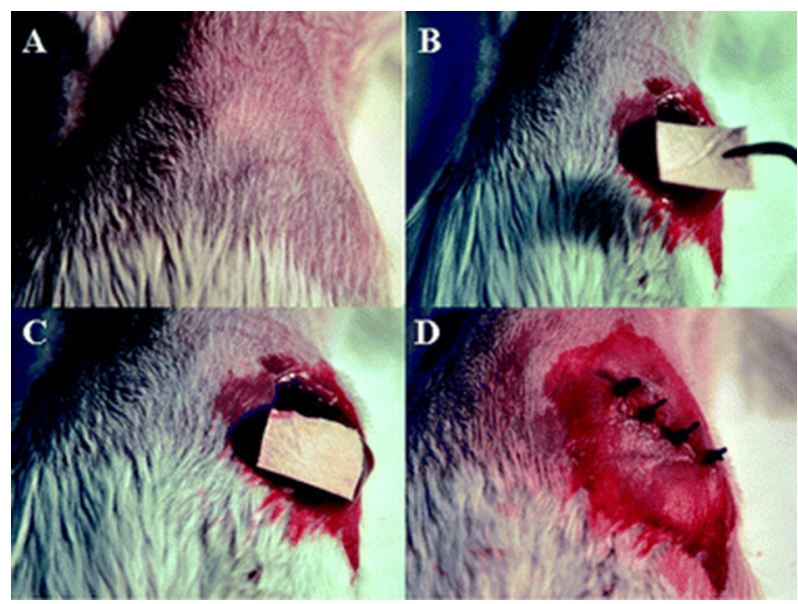

Fig. 6.4 Therapeutic process of nanofibers in the treatment of mice: (A) anesthetization of mice, (B) exposure of the liver after laparotomy, (C) direct placement of drug-loaded nanofibers on the tumor site, and (D) abdominal closure.

Reproduced with permission from Liu S, Zhou G, Liu D, Xie Z, Huang Y, Wang X, Wu W, Jing $\mathrm{X}$. Inhibition of orthotopic secondary hepatic carcinoma in mice by doxorubicin-loaded electrospun polylactide nanofibers. J Mater Chem 2013;B1: 101-9, with permission from Royal Society of Chemistry. 
bearing nodular SHCC (NSHCC). Seven days after the cell injection, whitish and irregular tumor nodules were observed in the liver lobe during explorative laparotomy of the mice as clearly seen in Fig. 6.5A. After the identification of NSHCC, a 6\% Dox fiber-mat of required size and shape was placed on the surface of the tumor nodule. Although the animals underwent second surgery within a week, they soon returned to their preoperative physical state. On day 30, all animals were still alive. Then, they were sacrificed and their livers were harvested. Macroscopic observation showed that most of the livers $(5 / 6)$ in Dox fibers group displayed the appearance of normal liver tissue except the tumor site covered by the fiber-mat (Fig. 6.5B), whereas the tumor nodule was big and took a quite fraction of the liver in the control group (Fig. 6.5C). Pasting of 6\% Dox fibers did cause a statistically marked reduction in tumor burden in the liver $(P<.01)$, the ratio of aspartate aminotransferase over alanine transaminase in serum (AST/ALT, a clinical indicator used in differentiating between causes of liver damage, $P<.01)$, and the AST activities $(P<.05)$ compared with those of the control group (Fig. 6.5D).

Jiang et al. [13] fabricated a three-dimensional aligned layer-by-layer nanofibrous scaffold by incorporation of poly ( $\varepsilon$-caprolactone)-poly(ethylene glycol) (PCE) copolymer nanofibers into porous chitosan $(\mathrm{CHI})$ to dictate the oriented regeneration of periodontium with aligned periodontal ligament (PDL) fibers perpendicular to the root surface. In vivo evaluation of the prepared scaffolds was conducted in rats (Sprague-Dawley) model having periodontal defects in four groups. The surgical process performed during the study is presented in Fig. 6.6. Two months following
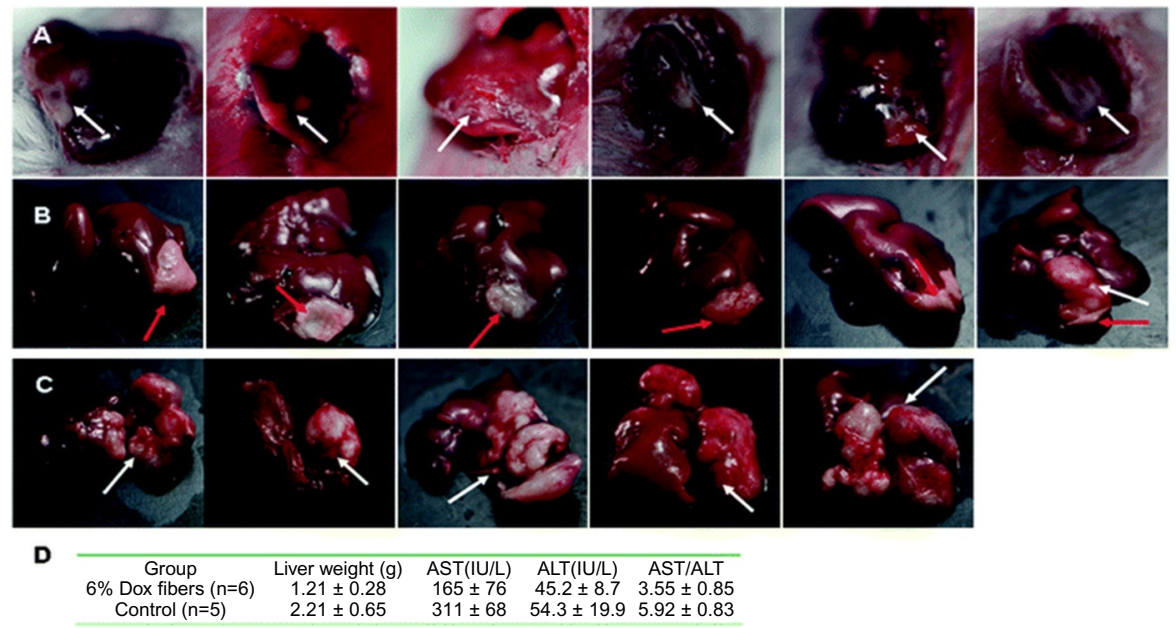

Fig. 6.5 Dox fibers inhibited NSHCC in Balb/c mice. Photographs of NSHCC before fiber-mat placement (A), 23 days after 6\% Dox fiber placement (B), and 30 days without any treatment (control) (C). The white arrow indicates the site of tumor and the red arrow indicates the site of Dox fiber-mat. (D) Antitumor effects of $6 \%$ Dox fibers in mice bearing NSHCC.

Reproduced with permission from Liu S, Zhou G, Liu D, Xie Z, Huang Y, Wang X, Wu W, Jing $\mathrm{X}$. Inhibition of orthotopic secondary hepatic carcinoma in mice by doxorubicin-loaded electrospun polylactide nanofibers. J Mater Chem 2013;B1: 101-9, with permission from Royal Society of Chemistry. 
(A)
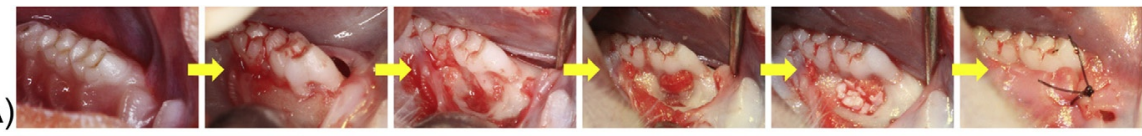

(B)
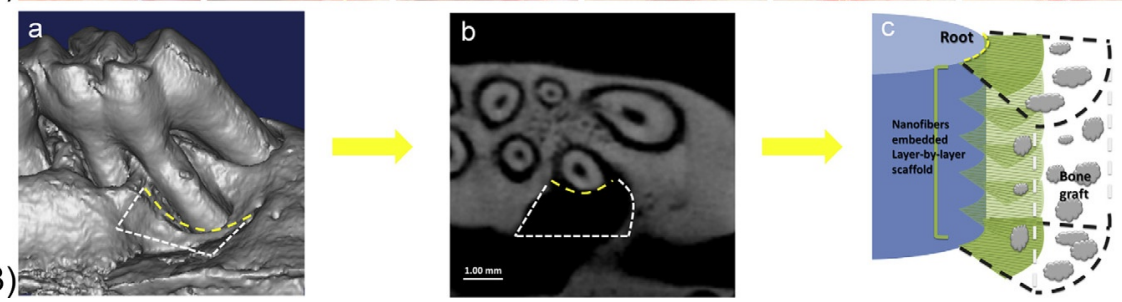

Fig. 6.6 Establishment of the rat periodontal defect model. (A) Surgical procedures of the periodontal fenestration defect at the maxillary first molar. (B) micro-CT reconstructed image scan (a), micro-CT transverse view (b), and schematic diagram (c) of the standardized defect with layers of fibers placed perpendicular against the root surface.

Reproduced with permission from Jiang W, Li L, Zhang D, Huang S, Jing Z, Wu Y, Zhao Z, Zhao L, Zhou S. Incorporation of aligned PCL-PEG nanofibers into porous chitosan scaffolds improved the orientation of collagen fibers in regenerated periodontium. Acta Biomater 2015;25:240-52, with permission from Elsevier.

the surgery, the ligament tissue organization from different groups was examined using hematoxylin and eosin (H\&E) staining as illustrated in Fig. 6.7. The histological images show that the distinct topological structures of three scaffolds led to different morphological patterns of PDL healing. The topographic guidance of scaffolds for regenerated tissue was strong. Disorderly and unsystematic regeneration was generated in blank control group, with irregular fibers along the root surface. The fibers in porous control group were more compact than that in blank control group but were still in random arrangement. In contrast, the PCE nanofiber-embedded scaffolds (i.e., 3D-RD and $3 \mathrm{D}-\mathrm{AL}$ ) presented much more defined orientation of regenerated tissues, as blank arrows with dash line indicated in Fig. 6.7 A(c and d) in which the fibers of 3D-AL were more parallel and PDL-like than that of 3D-RD. Moreover, cementum-like tissue layers were deposited on the dentin surfaces in 3D-AL scaffold, as the yellow arrows indicate. The developed scaffold has a great capacity of providing topographic cues and subsequently guiding the oriented regeneration of periodontal tissue in vivo.

\subsubsection{Clinical studies in human}

Despite many efforts have been paid in recent past for developing functional nanofibers to suit different biomedical applications, the studies in clinical phase are very limited and need further insight. Kossovich et al. [14] demonstrated the preparation of chitosan/ polyethylene oxide(PEO) nanofibers by electrospinning method, and clinical trial study was conducted in patients with II, IIIa, and IIIb degree burns. The nanofibrous membranes of thickness $200 \mu \mathrm{m}$ were applied onto burns, and their wound-healing behavior was studied. The clinical trial results indicated that chitosan nanofibrous dressing reduces the pain from dressing removal, absorption of exudate, ventilation of the wound, protection from infection, and further stimulates the process of skin tissue regeneration 

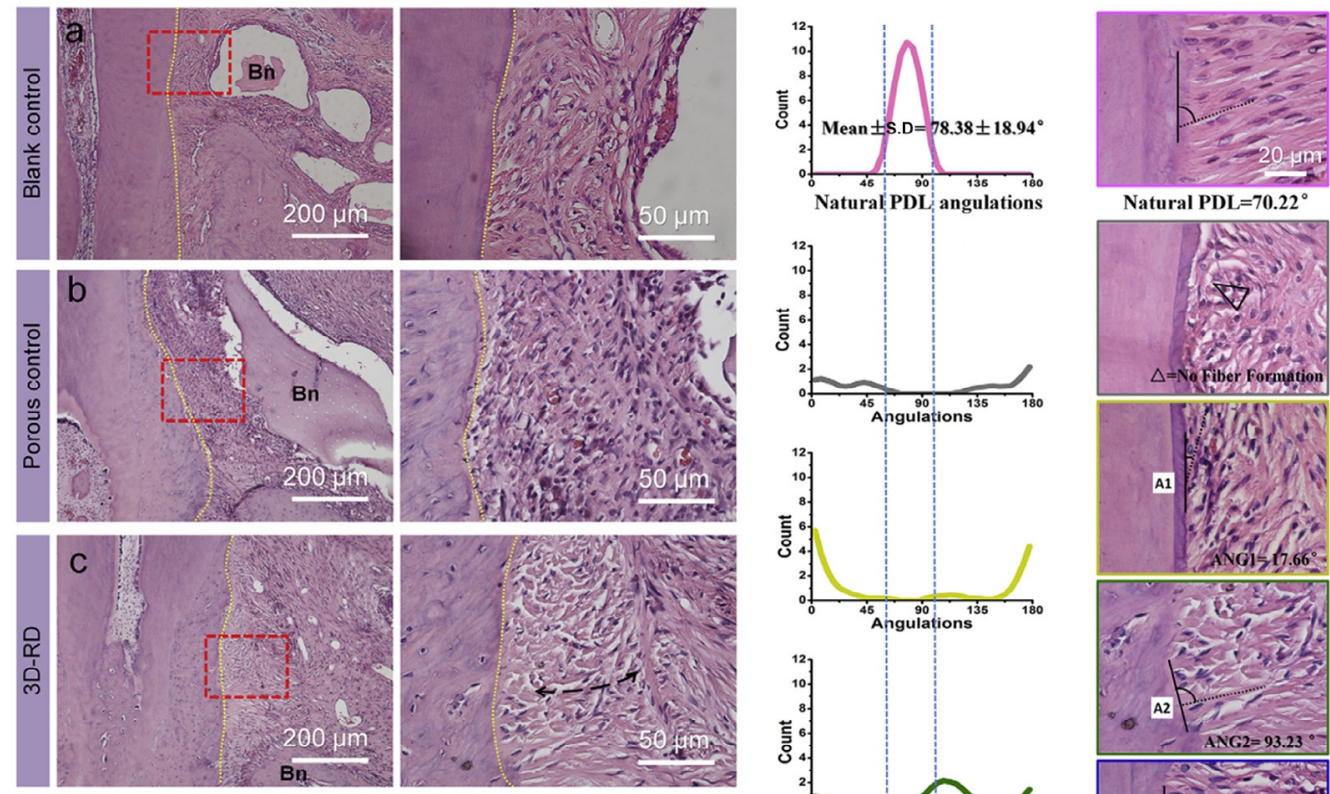

Natural PDL $=70.22^{\circ}$
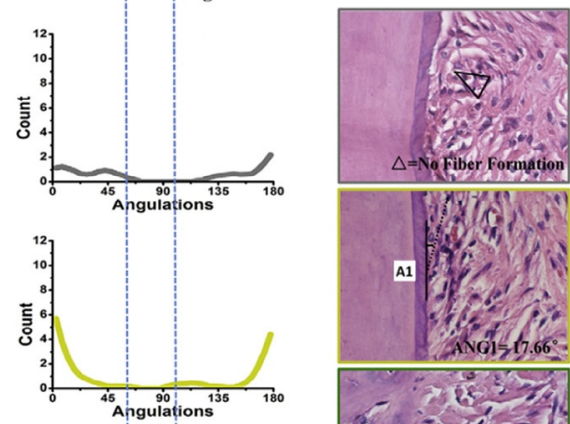

(A)
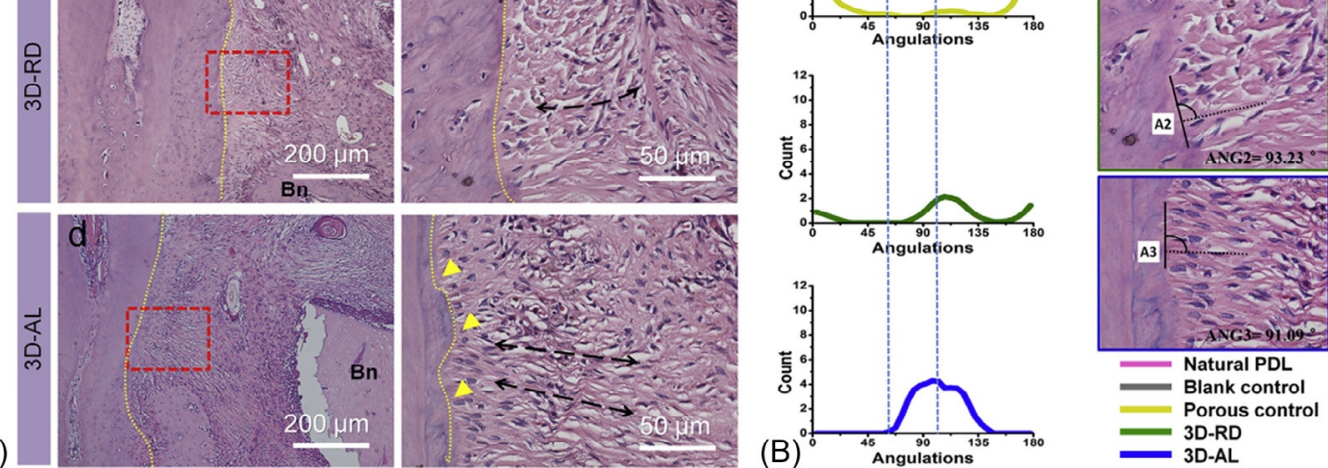

Fig. 6.7 In vivo-regenerated PDL-like tissue arrangement after 2 months. (A) Hematoxylin and eosin (H\&E) staining (a-d) to determine the arrangement of PDL-like fibrous connective tissue against the root surfaces. The images on the right are higher magnifications of images in the red frames on the left. Black dash lines indicated the orientation of regenerated PDL-like tissues. Yellow arrows indicate the newly formed cementum. Green arrows indicate the intensive collagen formation against the root surfaces. (B) The angular distribution of each experimental group, compared with the natural PDL ligament as a reference (average angular value $=8.38 \pm 18.94^{\circ}$ ), and the angular values of each representative image: natural PDL, no fiber formation, and angulated ligament tissues

Reproduced with permission from Jiang W, Li L, Zhang D, Huang S, Jing Z, Wu Y, Zhao Z, Zhao L, Zhou S. Incorporation of aligned PCL-PEG nanofibers into porous chitosan scaffolds improved the orientation of collagen fibers in regenerated periodontium. Acta Biomater 2015;25:240-52, with permission from Elsevier. 
(Figs. 6.8 and 6.9). Further, the degradation of this fibrous membrane prevents the mechanical damage of wound during removing dressings.

It is worth to note that few electrospun nanofibrous products are commercialized. The AVflo is a CE-certified electrospun nanofibrous-based polyurethane vascular access graft developed by Nicast Ltd, Israel, which is currently available in Asian countries, European Union market, and Israel [15]. The clinical trial studies of AVflo were conducted in patients and well reported [16]. The company is in further progress of developing nanofiber-based products such as nanostructured bioactive small-diameter vascular graft, biodegradable implant toward a better therapy for visual sensory impairments, biomimetic nanofiber-based nucleus pulposus regeneration for the treatment of degenerative disk disease, and NovaMesh Ventral Hernia Mesh.

Only few more efforts have been made to study the clinical effects of electrospun nanofiber-based products. The clinical trial website [17] was used to find out the list of clinical trials that were conducted on electrospun nanofiber-based products. The search results using the terms electrospinning and nanofibers are presented in Table 6.1. As summarized, the patches and nanofibers prepared by electrospinning technique were investigated in different phase studies to treat various conditions.

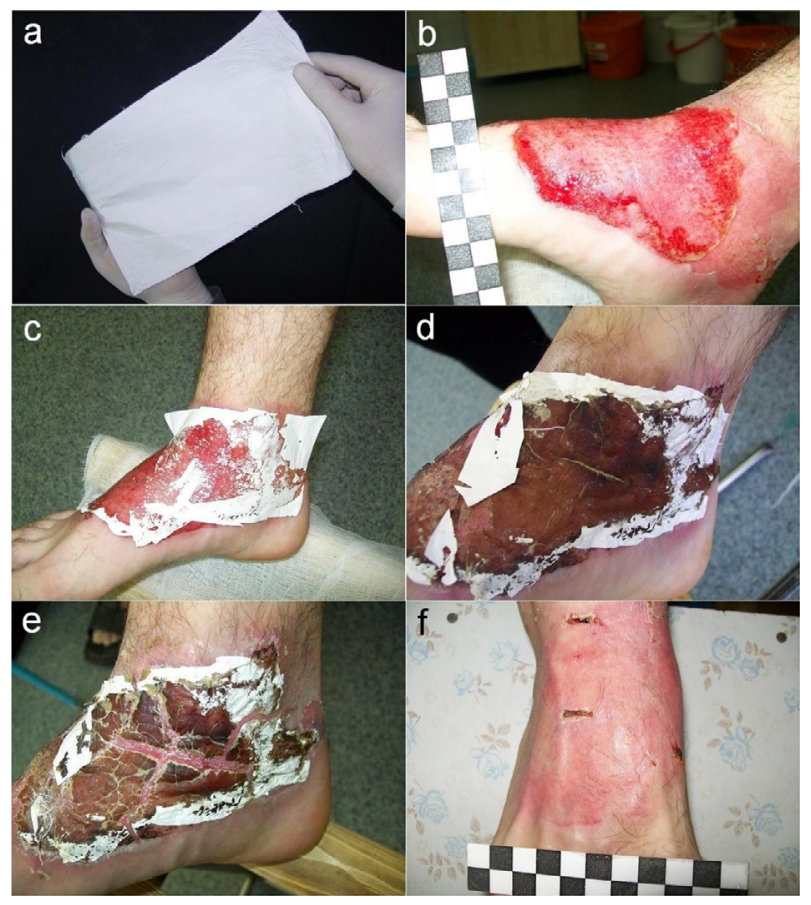

Fig. 6.8 Example of healing of IIIa burn: (A)sample of chitosan nanofiber dressing, (B) IIIa burn before covering, (C) after covering, (D) 5 days after covering, (E) 10 days after covering, and (F) 14 days after covering.

Reproduced with permission from Kossovich LY, Salkovskiy Y, Kirillova IV. Electrospun chitosan nanofiber materials as burn dressing. IFMBE Proc 2010;31:1212-14, with permission from Springer. 


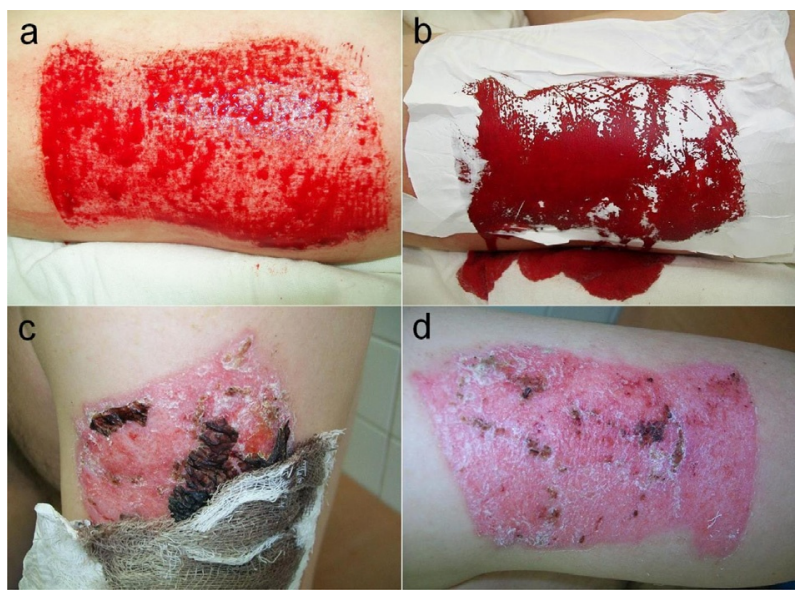

Fig. 6.9 Donor wound: (A) before covering, (B) after covering, (C) 12 days after covering, and (D) 14 days after covering.

Reproduced with permission from Kossovich LY, Salkovskiy Y, Kirillova IV. Electrospun chitosan nanofiber materials as burn dressing. IFMBE Proc 2010;31:1212-14, with permission from Springer.

Table 6.1 Summary of clinical trials conducted on electrospun nanofibers and patches

\begin{tabular}{|c|c|c|c|}
\hline $\begin{array}{l}\text { ClinicalTrials. } \\
\text { gov } \\
\text { Identifier: }\end{array}$ & Study title & Condition & Status \\
\hline NCT00317629 & $\begin{array}{l}\text { Controlled Nitric Oxide Releasing } \\
\text { Patch Versus Meglumine } \\
\text { Antimoniate in the Treatment of } \\
\text { Cutaneous Leishmaniasis }\end{array}$ & $\begin{array}{l}\text { Cutaneous } \\
\text { leishmaniasis }\end{array}$ & Terminated \\
\hline NCT00428727 & $\begin{array}{l}\text { Clinical Trial for the Treatment of } \\
\text { Diabetic Foot Ulcers Using a Nitric } \\
\text { Oxide Releasing Patch: PATHON }\end{array}$ & Diabetic foot & Completed \\
\hline NCT02237287 & $\begin{array}{l}\left.\text { Combination of Taliderm }{ }^{(}\right) \text {and } \\
\text { Vacuum-assisted Closure (VAC) for } \\
\text { Treatment of Pressure Ulcers }\end{array}$ & $\begin{array}{l}\text { Wounds } \\
\text { Pressure } \\
\text { ulcer }\end{array}$ & Terminated \\
\hline NCT02255188 & $\begin{array}{l}\text { Experimental Study of the Vascular } \\
\text { Prosthesis Manufactured by } \\
\text { Electrospinning }\end{array}$ & $\begin{array}{l}\text { Arterial } \\
\text { occlusive } \\
\text { disease }\end{array}$ & Ongoing \\
\hline NCT02680106 & $\begin{array}{l}\text { Evaluation of the SPINNER Device } \\
\text { for the Application of Wound } \\
\text { Dressing: Treatment of Split Skin } \\
\text { Graft Donor Sites (SPINNER01) }\end{array}$ & $\begin{array}{l}\text { Wound of } \\
\text { skin }\end{array}$ & Ongoing \\
\hline
\end{tabular}




\subsection{Conclusions and outlook}

In this chapter, we have presented an overview on the different types of in vivo studies and clinical trials conducted using electrospun nanofibrous materials, which are intended for various biomedical applications. To date, there are various hindrances in transferring lab-scale research to practical applications. The use of electrospun nanofibers in clinical applications is still in its infancy because of various issues including animal use, reliable correlation between in vitro-in vivo experiments, and high cost, which need to be addressed. In vivo testing of electrospun nanofibers is very few when compared with in vitro tests. However, most of the conducted in vivo studies in research laboratories are mainly not in accordance with standard safety guidelines to fulfill the requirements of the regulatory agencies. There is a great interest on generating toxicity data according to well-established regulatory guidelines [18], whereas there is no standard that specifically addresses the biological evaluation of medical devices containing nanomaterials till now. But, a new standard ISO 10993-Part 22 is under development for this purpose.

Considering the toxicity evaluation of electrospun nanofibers, factors including interaction of cells and nanoscaffolds, fiber diameter and pore size, surface patterning and topography, nanofiber alignment, fiber composition-wettability, and cellular response to electrospun scaffolds play critical role in study outcome. And also, it is recommended that designing of both in vitro and in vivo toxicity studies should follow the requirements of ISO 10993-Part 1:2010 standard in which the devices are classified under three main categories such as surface device (skin, mucosal membrane, breached, or compromised surface), external communicating device (blood path indirect, tissue/bone/dentin, and circulating blood), and implant device (tissue/bone and blood) based on their contact with the human body, directly or indirectly. In addition, the type of studies to be conducted is also based on their duration of exposure with the human body in which exposure duration is categorized as limited ( $\leq 24 \mathrm{~h})$, prolonged ( $24 \mathrm{~h}$ to 30 days), and permanent ( $>30$ days). Based on these factors, studies need to be conducted in line with established standards of ISO 10993. Most importantly, the FDA recommends that these studies need to be conducted in good laboratory practice (GLP) certified or ISO/IEC 17025-accredited laboratories.

\section{Acknowledgments}

B. B. acknowledges TUBITAK-BIDEB 2216, Research Fellowship Programme for Foreign Citizens for postdoctoral fellowship. A. S. acknowledges the Scientific and Technological Research Council of Turkey (TUBITAK), BIDEB 2221-Fellowships for Visiting Scientists, and Scientists on Sabbatical for the fellowship. T. U. acknowledges the Turkish Academy of Sciences-Outstanding Young Scientists Award Program (TUBA-GEBIP). 


\section{References}

[1] Zhang L, Webster TJ. Nanotechnology and nanomaterials: Promises for improved tissue regeneration. Nano Today 2009;4:6680.

[2] Cipitria A, Skelton A, Dargaville TR, Dalton PD, Hutmacher DW. Design, fabrication and characterization of PCL electrospun scaffolds-a review. J Mater Chem 2011;21:9419-53.

[3] ISO 14971:2007. Medical devices - Application of risk management to medical devices.

[4] ISO 10993-1:2010. Biological evaluation of medical devices - Part 1, Evaluation and testing within a risk management process.

[5] Goonoo N, Bhaw-Luximon A, Jhurry D. In vitro and in vivo cytocompatibility of electrospun nanofiber scaffolds for tissue engineering applications. RSC Adv 2014;4:31618-42.

[6] Ong CT, Zhang Y, Lim R, Samsonraj R, Masilamani J, Phan THH, et al. Preclinical evaluation of Tegaderm ${ }^{\mathrm{TM}}$ supported nanofibrous wound matrix dressing on porcine wound healing model. Adv Wound Care 2015;4:110-8.

[7] Hakimi O, Mouthuy PA, Zargar N, Lostis E, Morrey M, Carr A. A layered electrospun and woven surgical scaffold to enhance endogenous tendon repair. Acta Biomater 2015;26:124-35.

[8] Rosenman KD, Moss A, Kon S. Argyria: clinical implications of exposure to silver nitrate and silver oxide. J Occup Med 1979;21:430-5.

[9] Tseng YY, Kao YC, Liao JY, Chen WA, Liu SJ. Biodegradable drug-eluting poly [lactic-co-glycol acid] nanofibers for the sustainable delivery of vancomycin to brain tissue: in vitro and in vivo studies. ACS Chem Neurosci 2013;4:1314-21.

[10] Lowe A, Bills J, Verma R, Lavery L, Davis K, Balkus KJ. Electrospun nitric oxide releasing bandage with enhanced wound healing. Acta Biomater 2015;13:121-30.

[11] Liu S, Zhou G, Liu D, Xie Z, Huang Y, Wang X, et al. Inhibition of orthotopic secondary hepatic carcinoma in mice by doxorubicin-loaded electrospun polylactide nanofibers. J Mater Chem B 2013;1:101-9.

[12] Toshkova R, Manolova N, Gardeva E, Ignatova M, Yossifova L, Rashkov I, et al. Antitumor activity of quaternized chitosan-based electrospun implants against Graffi myeloid tumor. Int J Pharm 2010;400:221-33.

[13] Jiang W, Li L, Zhang D, Huang S, Jing Z, Wu Y, et al. Incorporation of aligned PCL-PEG nanofibers into porous chitosan scaffolds improved the orientation of collagen fibers in regenerated periodontium. Acta Biomater 2015;25:240-52.

[14] Kossovich LY, Salkovskiy Y, Kirillova IV. Electrospun chitosan nanofiber materials as burn dressing. IFMBE Proc 2010;31:1212-4.

[15] Nicast Homepage. http://www.nicast.com/index.aspx? [accessed 14.06.16].

[16] Wijeyaratne SM, Kannangara L. Safety and efficacy of electrospun polycarbonate-urethane vascular graft for early hemodialysis access: first clinical results in man. J Vasc Access 2011;12:28-35.

[17] Clinical Trials Homepage. http://www.clinicaltrials.gov/ [accessed 14.06.16].

[18] Brabu B, Haribabu S, Revathy M, Anitha S, Thangapandiyan M, Navaneethakrishnan KR, et al. Biocompatibility studies on lanthanum oxide nanoparticles. Toxicol Res 2015; 2015(4):1037-44. 